University of Nebraska - Lincoln

DigitalCommons@University of Nebraska - Lincoln

Faculty Publications, Department of Psychology

Psychology, Department of

$3-2010$

\title{
An Evaluation of the Applicability of the Tripartite Constructs to Social Anxiety in Adolescents
}

\author{
Emily R. Anderson \\ e.anderson@yale.edu \\ Glen J. Veed \\ University of Nebraska-Lincoln \\ Heidi M. Inderbitzen-Nolan \\ University of Nebraska-Lincoln \\ David J. Hansen \\ University of Nebraska-Lincoln, dhansen1@unl.edu
}

Follow this and additional works at: https://digitalcommons.unl.edu/psychfacpub

Part of the Psychiatry and Psychology Commons

Anderson, Emily R.; Veed, Glen J.; Inderbitzen-Nolan, Heidi M.; and Hansen, David J., "An Evaluation of the Applicability of the Tripartite Constructs to Social Anxiety in Adolescents" (2010). Faculty Publications, Department of Psychology. 506.

https://digitalcommons.unl.edu/psychfacpub/506

This Article is brought to you for free and open access by the Psychology, Department of at DigitalCommons@University of Nebraska - Lincoln. It has been accepted for inclusion in Faculty Publications, Department of Psychology by an authorized administrator of DigitalCommons@University of Nebraska - Lincoln. 


\title{
An Evaluation of the Applicability of the Tripartite Constructs to Social Anxiety in Adolescents
}

\author{
Emily R. Anderson \\ Yale University Child Study Center \\ Glen J. Veed, Heidi M. Inderbitzen-Nolan, and David J. Hansen \\ University of Nebraska-Lincoln \\ Corresponding author - Emily R. Anderson, Yale University Child Study Center, \\ 230 South Frontage Road, New Haven, CT 06520; email e.anderson@yale.edu
}

\begin{abstract}
The current study examined the tripartite model of anxiety and depression in relation to social phobia in a nonclinical sample of adolescents (ages 13-17). Adolescent/parent dyads participated in a semistructured interview and completed self-report measures of the tripartite constructs and social anxiety. Adolescents gave an impromptu speech, and heart rate was monitored. Low positive affect, high negative affect, and high physiological hyperarousal were characteristic of adolescents diagnosed with social phobia; adolescents with elevated social anxiety symptoms who did not meet criteria for social phobia did not evidence low positive affect. Heart rate reactivity during the speech was not significantly correlated with social anxiety symptomatology or with self-reported physiological hyperarousal.
\end{abstract}

Es: xtensive research indicates that anxiety and depression overlap in youth, as evidenced by high intercorrelations of self-report measures of anxiety and depression and by high rates of comorbid anxiety and depressive disorders. The shared variance between these two symptom clusters has been estimated to be as high as $72 \%$ (Cole, Truglio, \& Peeke, 1997) and controlling for item overlap between measures has resulted in only a minimal decrease in shared variance (Cole et al., 1997; Stark \& Laurent, 2001). In a review of the literature, Brady and Kendall (1992) found a 16\% comorbidity rate for anxiety and depressive disorders in community, non-treatment-seeking youth samples and rates ranging from $28 \%$ to $62 \%$ in clinical samples. Correlations are substantially high between social phobia and depression. In fact, epidemiological studies indicate that $25 \%$ to $31 \%$ of youth with social anxiety have been diagnosed with or exhibit symptoms of comorbid depression (Essau, Conradt, \& Petermann, 1999; Last, Strauss, \& Francis, 1987; Wittchen, Stein, \& Kessler, 1999) and rates in clinical samples are even higher, ranging from $17 \%$ to $52 \%$ (Last, Perrin, Hersen, \& Kazdin, 1992).
Clark and Watson (1991) proposed a tripartite model to account for the observed overlap between anxiety and depressive symptoms, as well as to explain high comorbidity rates. This model posits that anxiety and depression share a common component of negative affect (NA), which accounts for symptom overlap and comorbidity. NA was proposed to represent high objective distress. Substantial correlations between self-report measures of anxiety and depression support the notion that they tap into an underlying construct of NA. Clark and Watson hypothesized that anxiety and depression can be differentiated by positive affect (PA) and physiological hyperarousal (PH). PA was proposed to represent pleasurable engagement with the environment. $\mathrm{PH}$ was suggested to include somatic tension, shortness of breath, dizziness, dry mouth, and panic, including cardiovascular symptoms. Clark and Watson found that low PA is unique to depression, whereas high PH characterizes anxiety disorders.

Empirical research has supported the utility of the tripartite model for explaining the association between anxiety and depression in children and adolescents. 
Several investigations have supported two-factor models of PA and NA as distinct constructs in school children (Cole et al., 1997; Crook, Beaver, \& Bell, 1998; Stark \& Laurent, 2001) and adolescents (Huebner \& Dew, 1995; Inderbitzen \& Hope, 1995; Lonigan, Hooe, David, \& Kistner, 1999; Valentiner, Gutierrez, \& Blacker, 2002). More recent research has supported the utility of all three constructs in children and adolescents in clinical (Joiner, Catanzaro, \& Laurent, 1996; Joiner \& Lonigan, 2000; Lonigan, Carey, \& Finch, 1994) and nonclinical (Cannon \& Weems, 2006; Olino, Klein, Lewinsohn, Rohde, \& Seeley, 2007; Phillips, Lonigan, Driscoll, \& Hooe, 2002) settings.

Several critics (Brown, Chorpita, \& Barlow, 1998; Chorpita, Albano, \& Barlow, 1998; Watson, Gamez, \& Simms, 2005) have noted that a major limitation of Clark and Watson's tripartite model is its failure to account for substantial heterogeneity among the anxiety disorders. Other research has only partially supported the tripartite model across the anxiety disorders and, specifically, in social phobia. Research with adults (Watson, Clark, \& Carey, 1988; Watson et al., 2005) and youth (Chorpita, Plummer, \& Moffitt, 2000) found that NA is not elevated in individuals with social phobia, contrary to the original model. Evidence from studies with adults and children suggests that low PA is associated with social phobia (Brown et al., 1998; Chorpita et al., 2000; Watson, Clark, \& Carey, 1988; Watson et al., 2005), also contrary to the original model. One explanation for this finding is that PA is negatively correlated with frequency and satisfaction of contact with friends, ability to make new friends, and involvement in social organizations (Watson, Clark, \& Carey, 1988). The unique relationship of social phobia and low PA may be related to the interpersonal character of low PA, including low confidence and unassertiveness, which could explain why low PA is not related to the other anxiety disorders (Brown et al., 1998).

Several studies also have suggested that, contrary to the original model, $\mathrm{PH}$ is most significantly associated with panic disorder and is not related to social phobia in adults (Brown et al., 1998; Joiner et al., 1999) or in children (Chorpita et al., 1998). Clark and Watson's definition of the PH construct, which includes autonomic hyperactivity, has been problematic to measure and typically has been assessed through self-report (Greaves-Lord et al., 2007). Only low to moderate correlations, however, have been found between subjective and objective arousal during social-evaluative situations (Mauss, Wilhelm, \& Gross, 2004). No research has explored this relationship in youth. Limited research has examined the relationship between subjective and objective arousal, including heart rate (HR) reactivity. One investigation used an objective measure of $\mathrm{PH}$ and found no relationship between self-reported anxiety and resting heart rate in a community sample of young ad- olescents (Greaves-Lord et al., 2007). A significant positive relationship was found, however, between resting heart rate and internalizing symptoms. Examination of objective arousal, including heart rate, in youth with specific anxiety disorders was recommended. To date, no research has investigated the relationship between an objective physiological measurement of $\mathrm{PH}$ and social phobia, despite a recommendation to do so (Laurent \& Ettelson, 2001). Last, it is unclear if the PH construct represents a broad emotional construct or if it is situation specific. Objective measurement of $\mathrm{PH}$ in an anxiety-provoking situation could provide a better understanding and definition of the PH construct in youth and potentially improve discriminant validity for anxiety diagnoses, including social phobia.

Moving toward a disorder-specific evaluation of the tripartite model, one recent study investigated the tripartite constructs in adults diagnosed with social phobia (Hughes et al., 2006). Results suggested that the nature of social phobia symptoms is differentially related to the tripartite constructs. Self-reported social anxiety was associated with low PA but not with high $\mathrm{PH}$, after controlling for general distress. Individuals with generalized social phobia, however, exhibited low PA, whereas individuals with performance anxiety exhibited high PH. Thus, nongeneralized social phobia may be more closely related to the anxiety disorders, whereas generalized social phobia may be more similar to the mood disorders.

Evidence indicates that the tripartite constructs may function differently in social phobia than proposed by the original model. However, less research has examined the tripartite constructs in children and adolescents with social phobia, and thus further evaluation is warranted (Anderson \& Hope, 2008). Adolescence is an ideal developmental time frame in which to examine the tripartite constructs in relation to social phobia, because onset typically occurs during this period. Given that adults with social phobia are at least four times more likely to develop depression compared to individuals without social phobia (Moutier \& Stein, 1999), there may be a developmental progression from social phobia to depression. Earlier identification and treatment may prevent later morbidity and associated impairment. As noted by Weems and Stickle (2005), adequate inclusion of the tripartite constructs in the definitions of anxiety/ depressive disorders may help with discriminant validity for diagnoses.

The purpose of the present study was to evaluate the tripartite constructs in social phobia in a nonclinical sample of adolescents. The current study is significant because it addresses several limitations from previous research. First, symptom prevalence and severity were assessed through self-report measures and a semistructured diagnostic interview. Thus, the sample was comprised of adolescents exhibiting a continuum of 
symptoms, which allowed for examination of the tripartite constructs across differing severity levels of social anxiety. Second, we used scales that have been empirically supported to represent the constructs of PA and NA (Positive and Negative Affect Scale [PANAS]; Watson, Clark, \& Tellegen, 1988), as well as selected items from the Beck Anxiety Inventory (Beck, Epstein, Brown, \& Steer, 1988) that were identified as reliable measurements of PH (Joiner et al., 1999). Third, this investigation utilized an objective measure of $\mathrm{PH}$, which has not been adequately evaluated (Laurent \& Ettelson, 2001) and which facilitated exploration of the relationship between subjective and objective $\mathrm{PH}$.

Several hypotheses were made based on the observed differential functioning of the tripartite constructs in social phobia (Brown et al., 1998; Chorpita et al., 1998; Chorpita \& Daleiden, 2002; Chorpita et al., 2000; Joiner et al., 1999; Watson, Clark, \& Carey, 1988; Watson et al., 2005). First, it was hypothesized that social anxiety would be significantly negatively correlated with PA but not correlated with NA or PH. Second, it was hypothesized that adolescents classified as socially anxious (described next) and adolescents diagnosed with social phobia would exhibit lower mean scores on the PA construct compared to nonanxious adolescents. It was furthermore hypothesized that adolescents in the social phobic group would exhibit lower mean scores on the PA construct compared to adolescents in the socially anxious group, given that the former group, by their diagnostic status, experiences more impairment. In addition, it was hypothesized that there would be no differences among the three groups with regard to mean scores on the constructs of NA or PH. Last, analyses were conducted to examine the relationship between HR reactivity and social anxiety and between $\mathrm{HR}$ reactivity and self-reported $\mathrm{PH}$; however, no a priori hypotheses were made, given the paucity of previous research.

\section{Method}

\section{Participants}

One hundred seventy adolescents and their parents were recruited from middle and high schools in a small Midwestern city, as part of a larger study. The sample comprised 81 boys and 89 girls between the ages of 13 and 17 ( $M$ age $=14.7)$. The ethnic background of participants was representative of the larger community as $87.6 \%$ of the sample was Caucasian, 4.1\% was African American, $0.6 \%$ was Asian American, $2.9 \%$ was Hispanic, $2.4 \%$ was Native American, and $2.4 \%$ was biracial. Participants in the study reported the following breakdown of socioeconomic statuses: family income less than $\$ 10,000$ (1.2\%), $\$ 11,000$ to $\$ 25,000$ (18.8\%), $\$ 26,000$ to $\$ 50,000(29.4 \%), \$ 51,000$ to $\$ 75,000(27.1 \%), \$ 76,000$ to $\$ 100,000(14.7 \%)$, and greater than $\$ 100,000$ (5.3\%). With regard to participants' current living situation, 58.8\% reported living with both parents, $38.2 \%$ reported living only with their mother, and $0.6 \%$ reported living only with their father $(2.4 \%$ of participants did not report their living situation).

\section{Measures}

Anxiety Disorders Interview Schedule for DSMIV: Child \& Parent Versions (ADIS-IV: C/P; Silverman \& Albano, 1996) - The ADIS-IV: C/P is a semistructured diagnostic interview that is organized diagnostically according to anxiety disorders in the $\mathrm{Di}$ agnostic and Statistical Manual of Mental Disorders (4th ed. [DSM-IV]; American Psychiatric Association, 1994) and assesses for Dysthymia, Major Depressive Disorder, Attention Deficit/Hyperactivity Disorder, Conduct Disorder, and Oppositional Defiant Disorders. It has proven to be highly reliable in assessing anxiety disorder diagnoses (e.g., Wood, Piacentini, Bergman, McCracken, \& Barrios, 2002). The ADIS-IV: C/P is administered separately to the adolescent and the parent and independent diagnoses are made for each interview. Each diagnosis is augmented with a clinician's severity rating (CSR), based on impairment in functioning and symptom severity. CSRs range from 0 (none) to 8 (very severely disturbing/disabling), and a minimum rating of four is required to make a diagnosis. The ADIS-IV: C/P provides three separate diagnoses: one from the adolescent interview, one from the parent interview, and a composite diagnosis. The composite diagnosis includes all diagnoses from both adolescent and parent interviews. If the adolescent and parent interview result in the same diagnosis, the higher of the CSRs is applied to the composite diagnosis. In the present study the composite diagnosis, in conjunction with two self-report measures, was used to form groups described next.

All diagnostic interviews were completed by trained doctoral-level clinical graduate students. All interviewers were trained based on the criteria outlined by the authors of the ADIS-IV: C/P (Silverman \& Albano, 1996). To assess for independent diagnostic agreement, all diagnostic interviews were audio-taped. A second trained interviewer randomly selected and reevaluated $25 \%$ of the tapes to assess for interrater reliability. It was considered a diagnostic match if there was exact agreement on the composite diagnoses and CSRs were within 1 point, similar to the interviewer training process. CSRs which differed by 1 point were not considered a match at the threshold of diagnosis (i.e., CSRs of 3 and 4), given that the latter resulted in a diagnosis but the former did not. In the present sample, interrater agreement 
was found to be $94 \%$ overall and $100 \%$ for social phobia diagnoses. All of the adolescents in the present sample met the 6-month duration criteria for a diagnosis of social phobia.

PANAS (Watson, Clark, \& Tellegen, 1988) - The PANAS is a 20-item inventory assessing two primary mood dimensions: positive affect (PANAS-P; 10 items such as interested, excited, proud) and negative affect (PANAS-N; 10 items such as upset, ashamed, nervous). Respondents indicate to what extent they have experienced each specific emotion in the past week. Each item is rated on a 5-point Likert-type scale ranging from 1 (very slightly or not at all) to 5 (very much or extremely). Scores for each scale range from 10 to 50, with higher scores indicating higher levels of positive and negative affect. There is considerable support for the construct validity of the PANAS (Watson, Clark, \& Tellegen, 1988), and estimates of internal consistency range from .86 to .90 for the PANAS-P and from .84 to .97 for the PANAS-N. The PANAS has been validated to measure the PA and NA constructs (e.g., Huebner \& Dew, 1995; Watson, Clark, \& Tellegen, 1988). Although the PANAS initially was developed for use with adults, Huebner and Dew supported its utility in adolescents. In the present investigation the PANAS$P$ evidenced good internal consistency $(\alpha=.76)$, as did the PANAS-N $(\alpha=.68)$. The PANAS scales were utilized in the present study as measures of positive and negative affect.

Beck Anxiety Inventory (BAI; Beck et al., 1988) - The BAI is 21-item self-report measure assessing severity of anxiety symptoms. Items are rated on a 4-point Likert-type scale ranging from 0 (not at all) to 3 (severely, I could barely stand it). Examples of BAI items include the following: "feeling hot," "wobbliness in the legs," "dizzy or lightheaded," "heart pounding or racing," "difficulty breathing," and "face flushed." Confirmatory factor analyses have identified six specific items from the BAI (Items 2, 3, 6, 7, 15, and 20) consistent with the $\mathrm{PH}$ construct and with good discriminant validity with subjective anxiety, as well as positive and negative affect (Joiner et al., 1999). These six items were used to create the PH scale to measure $\mathrm{PH}$. The standard instructions for the BAI were altered slightly in that adolescents were asked to rate these symptoms during a speech task immediately prior rather than over the past week. In the present investigation the PH scale evidenced excellent internal consistency $(\alpha=.87)$.

Social Anxiety Scale for Adolescents (SAS-A; La Greca \& Lopez, 1998; La Greca \& Stone, 1993) The SAS-A is an 18-item self-report measure assess- ing fear of negative evaluation, social avoidance, and distress. Items are rated on a 5-point Likert-type scale ranging from 1 (not at all true) to 5 (true all of the time). Higher scores indicate higher social anxiety. Examples of the items include "I worry about being teased" and "It's hard for me to ask others to do things with me." The SAS-A has demonstrated good internal consistency, and there is evidence to support its concurrent and construct validity (Inderbitzen-Nolan \& Walters, 2000). In the present investigation the SAS-A evidenced excellent internal consistency $(\alpha=.90)$. The suggested cutoff score to reliably differentiate socially anxious and nonanxious adolescents is 50 (La Greca \& Lopez, 1998). Using a larger set of data from the current project, Inderbitzen-Nolan, Davies, and McKeon (2004) found that the specificity of the SAS-A was quite high $(82.7 \%)$, whereas the sensitivity was only $43.6 \%$. For the purposes of the present study, a method was needed to identify adolescents who endorsed elevated social anxiety symptomatology but did not meet criteria for a disorder, and the cutoff score of 50 was used for this purpose. The SAS-A was included in the present study to assist in group formation and as a measure of social anxiety.

Social Phobia and Anxiety Scale for Children (SPAI-C; Beidel, Turner, \& Morris, 1995) - The SPAI$\mathrm{C}$ is a self-report measure that includes 26 items assessing somatic, cognitive, and behavioral symptoms associated with social phobia. Each item is responded to on a 3-point Likert-type scale (never or hardly ever, sometimes, almost always or always) assessing the frequency of anxious feelings across various situations. Higher scores are indicative of higher anxiety. Examples of items include "I feel scared when I have to speak or read in front of a group of people" and "I am too scared to ask questions in class." Beidel and colleagues (1995) found good test-retest reliability $(r=.86)$ and good internal consistency $(\alpha=.95)$ for the SPAI-C. There is also has evidence to support the concurrent, convergent, and discriminant validity of the SPAI-C (Beidel, Turner, \& Fink, 1996; Morris \& Masia, 1998). In the present investigation, the SPAI-C evidenced excellent internal consistency ( $\alpha=.97)$. Several studies support the suggested cutoff score of 18 to reliably differentiate socially anxious and non-socially anxious children (Beidel et al., 1995; Beidel, Turner, \& Morris, 1998) and to differentiate children with social phobia from children with other anxiety disorders (Beidel, Turner, Hamlin, \& Morris, 2000). Inderbitzen-Nolan and colleagues (2004) found that the specificity of the SPAI-C was quite high $(82.7 \%)$, and the sensitivity was $61.5 \%$. The SPAI-C was included in the present study to assist in group formation and as a measure of social anxiety. 
Anxiety-provoking task - Each participant gave a 10-min impromptu speech in front of an audience of three (one graduate student and two undergraduate students), which was videotaped. Immediately prior to the speech, the adolescent was given a list of five possible topics to discuss and $3 \mathrm{~min}$ to prepare for the speech but was not allowed to make any notes. The participant was instructed to speak about any of the topics on the list and was given the option to talk about topics not included on the list. If the adolescent stopped talking or asked to stop the speech, he or she was asked to continue; however, if this occurred twice, the participant was allowed to stop. The speech also was discontinued if the adolescent did not speak for a full minute. No adolescents refused to give the speech, and the speech was discontinued early for 20 adolescents. Just prior to and immediately following the speech adolescents rated their Subjective Units of Distress (SUDS) on a scale of 0 to 8 , indicating anxiety before the speech and highest anxiety experienced during the speech, respectively. The speech task was chosen because the most commonly endorsed anxiety-provoking social situation in adolescence is public speaking (Beidel \& Randall, 1994). In the current sample, $75 \%$ of adolescents diagnosed with social phobia endorsed a speech task as highly anxiety-provoking on the ADIS-IV: C/P (Smith, Merritt, \& Inderbitzen-Nolan, 2004).

Heart rate measurement - Participants' heart rate was assessed every $5 \mathrm{sec}$ through a portable heart rate monitor on their chests (Vantage NV, Polar, Lake Success, NY). Heart rate was later downloaded onto a computer (using the Advantage Interface System, Polar, Lake Success, NY) and computed as beats per minute. Heart rate was then averaged across each minute of the baseline period and each minute of the speech. An average baseline value was calculated for the 10 min prior to the speech. Change scores were calculated by subtracting average baseline values from heart rate measurements for each minute during the speech to examine reactivity during the speech. Thus, positive change scores indicated an increase in heart rate during the speech. Previous studies have successfully utilized portable heart rate monitors with social phobic participants (Gerlach, Wilhelm, \& Roth, 2003; Grossman, Wilhelm, Kawachi, \& Sparrow, 2001; Heimberg, Dodge, Hope, Kennedy, \& Zollo, 1990; Heimberg, Hope, Dodge, \& Becker, 1990).

\section{Procedures}

All measures were collected as part of a larger research study funded by National Institute of Mental Health. All of the study procedures were approved by the Institutional Review Board. Recruitment let- ters were mailed to parents of adolescents in Grades 7 through 12 in public middle and high schools. These letters described a research project focused on adolescents who reported being shy or feeling anxious in social situations. The letters stated that youth who did not report such feelings also were needed, and thus, any youth between the ages of 13 and 17 may be eligible for participation. Parents completed an initial phone screen to determine eligibility. For the purposes of the larger study, the following exclusion criteria, per parent report, were used: diagnoses of learning disabilities and treatment histories for Bipolar Disorder, Major Depressive Disorder, Attention Deficit/Hyperactivity Disorder, Conduct Disorder, and Oppositional Defiant Disorders.

Over the course of the first 2 years of the 5-year study from which the current data were collected, approximately 4,050 letters were mailed to parents and guardians. Telephone calls requesting additional information about the study were received from approximately 280 parents or adolescents $(6.9 \%$ of those purportedly receiving flyers). Appointments were scheduled with 207 adolescent-parent pairs (74\% of those calling), and 170 adolescent-parent pairs ( $82 \%$ of those who had a scheduled appointment) actually attended the assessment appointment.

If the adolescent met the inclusion criteria after the phone screen, the parent and adolescent pair were scheduled for a first appointment. At the beginning of this appointment, the parent gave his or her consent to participate as well as consent for his or her child to participate. Assent was obtained from adolescents younger than age 18. The adolescent and parent then were interviewed separately using the ADIS-IV: C/P. Adolescents completed the PANAS, SAS-A, and SPAI$\mathrm{C}$ during this appointment. Adolescents were invited to return approximately 1 week later to participate in a second appointment. Because of the purposes of the larger study, two adolescents were not invited to return for a second appointment because they received a principal diagnosis of Dysthymia and Attention Deficit/Hyperactivity Disorder, respectively. At the beginning of the second appointment, the parent again gave consent, and the adolescent assented to participation. At this time adolescent participants were required to stand for the remainder of the appointment to control for postural changes that could impact reactivity, and heart rate was recorded for a 10-min baseline period prior to the impromptu speech task. After the baseline period, adolescents were informed about the nature of the task and were given 3 min to prepare for the speech; heart rate was not recorded during these $3 \mathrm{~min}$. Heart rate was monitored during the speech task, and subsequently adolescents rated their SUDS and completed the BAI. 


\section{Results}

\section{Formation of Diagnostic Groups}

Three groups were formed using the ADIS-IV: C/P and the two social anxiety self-report measures (i.e., SAS-A and SPAI-C): a social phobic group, a socially anxious group, and a nonanxious group. As previously mentioned, these groups were created to examine a continuum of social anxiety severity. Three groups were chosen because a significant number of adolescents (approximately one third) in the current sample reported clinically significant levels of social anxiety but did not receive a formal diagnosis of social phobia. These "socially anxious" youth seemed to represent a unique group.

Adolescents who received a principal composite diagnosis of social phobia with a CSR of 4 or greater were included in the social phobic group. This criteria resulted in 56 adolescents (26 boys, 30 girls) being classified as social phobic. Six of these adolescents were classified with nongeneralized social phobia, and the remaining 50 adolescents were diagnosed with generalized social phobia. Of the social phobic group, 33 participants had a principal diagnosis of social phobia and no other diagnoses, and 23 adolescents had a principal diagnosis of social phobia along with a secondary comorbid anxiety disorder (e.g., 16 had a comorbid Generalized Anxiety Disorder diagnosis and 7 had a Specific Phobia diagnosis). It should be noted that principal and secondary diagnoses were determined by the CSRs (i.e., the diagnosis with the highest CSR was the principal diagnosis).

One hundred two adolescents (51 boys, 51 girls) did not meet ADIS-IV: C/P diagnostic criteria for any anxiety, mood, or externalizing disorder and were further divided into two groups for the analyses. The nonanxious group was comprised of 45 adolescents (19 boys, 26 girls) who did not score above the cutoff scores on either the SAS-A (cutoff of 50; La Greca \& Lopez, 1998) or the SPAI-C (cutoff of 18; Beidel et al., 1995). The socially anxious group was comprised of 57 adolescents (32 boys, 25 girls) who scored above the cutoff on at least one of the social anxiety measures. We required scoring above the cutoff on at least one measure because, although there is significant overlap between the SAS-A and SPAI-C $(r=.75$ in the present study), they measure the construct in subtly different ways (Inderbitzen-Nolan et al., 2004). Previous research suggests that different youth score above the clinical cutoffs on each measure (Epkins, 2002; Morris \& Masia, 1998). Thus, to be conservative and to more completely capture the general construct of social anxiety in our sample, we chose to use cutoffs on either the SAS-A or SPAI-C in determining the socially anx- ious group. Of note, statistical analyses were conducted using adolescents who scored above the cutoff scores on both the SAS-A and SPAI-C resulted in findings similar to those presented here. Thirty-eight adolescents scored above the clinical cutoff on the SASA only, 1 adolescent scored above the clinical cutoff on the SPAI-C only, and 18 adolescents scored above the clinical cutoffs on both measures. The socially anxious and social phobic groups scored significantly higher than the nonanxious group on the SAS-A, F(2, $152)=58.55, p<.001$ (least significant difference (LSD) minimum mean difference $=4.85$ ) but were not significantly different from each other. On the SPAI-C, the social phobic group scored significantly higher than the socially anxious group, and the socially anxious group scored significantly higher than the nonanxious group, $F(2,151)=56.33, p<.001(\mathrm{LSD}$ minimum mean difference $=4.90$ ). Ten of the original sample of 170 adolescents were excluded prior to group formation due to having principal diagnoses of Generalized Anxiety Disorder $(n=5)$ and Specific Phobia $(n=5)$. As noted, 2 additional adolescents were not invited to return for a second appointment and thus were excluded prior to group formation. All subsequent analyses included 158 adolescents across the three groups.

\section{Preliminary Analyses}

Preliminary analyses were conducted to ensure that the three groups were equivalent with regard to demographic variables. Results from these analyses indicated that the groups were not significantly different on the following variables: age, $F(2,155)=2.29, p>.05$; gender, $\chi^{2}(2)=2.13, p>$.05; race, $\chi^{2}(10)=6.83, p>$.05; family income, $\chi^{2}(10)=7.56, p>.05$; and living situation, $\chi^{2}(6)=1.89, p>.05$ (see Table 1). Results presented in this article were not altered significantly by considering boys and girls separately, and therefore all analyses are conducted without considering gender as a covariate and without separating boys and girls.

Means, standard deviations, and indices of skewness and kurtosis were calculated for the PANASN, PANAS-P, PH scale, SAS-A, SPAI-C (see Table 2 for means and standard deviations), and each of the heart rate change scores for the speech (see Table 3 for means and standard deviations) to ensure that all variables were normally distributed. Outlier analyses were performed on each variable, and no outliers were detected. In addition, participants with more than two missing heart rate measurements $(n=39)$, due to a malfunctioning of the heart rate equipment, were not included in the analyses, which resulted in heart rate data being available from 119 participants. Data from participants who ended the speech early $(n=20)$ were retained. 
Table 1. Descriptive Characteristics for the NA, SA, and SP Groups

\begin{tabular}{lrrrr}
\hline Variable & NA $n$ & SA $n$ & SP $n$ & \multicolumn{1}{c}{$\chi^{2}$} \\
\hline Gender & & & & $2.13(n s)$ \\
$\quad$ Female & 26 & 25 & 30 & \\
$\quad$ Male & 19 & 32 & 26 & $6.83(n s)$ \\
Ethnicity & & & & \\
$\quad$ Caucasian & 36 & 52 & 49 & \\
Asian American & 0 & 0 & 1 & \\
Native American & 1 & 1 & 2 & \\
African American & 3 & 2 & 2 & \\
Hispanic & 2 & 1 & 1 & \\
Biracial & 2 & 0 & 1 & \\
Missing & 1 & 1 & 0 & \\
Annual Family Income & & & & \\
Under 10 K & 0 & 1 & 1 & \\
11-25 K & 13 & 8 & 9 & \\
26-50 K & 9 & 17 & 19 & \\
51-75 K & 14 & 14 & 17 & \\
76-100 K & 6 & 10 & 7 & \\
Over 100 K & 2 & 4 & 2 & \\
Missing & 0 & 3 & 1 & \\
Living Situation & & & & \\
Both Parents & 26 & 33 & 33 & \\
Mother Only & 18 & 22 & 21 & \\
Father Only & 1 & 0 & 1 & \\
Other & 0 & 1 & 1 & \\
OA & & &
\end{tabular}

$\mathrm{NA}=$ nonanxious group; $\mathrm{SA}=$ socially anxious group; $\mathrm{SP}=$ social phobic group; $\mathrm{ns}=$ not significant at $p<.05$.

To verify that the speech task was sufficiently anxiety provoking, participants' SUDS ratings were compared before and during the task. SUDS ratings were higher for all three groups during the speech than before the speech; however, a significant interaction of group and time (before and after the speech), $F(2$,
$133)=5.15, p<.01(\mathrm{LSD}=.52)$, indicated that the social phobic group reported a larger increase than the other two groups (see Table 2 for group means at each time point). To determine if the speech task was differentially anxiety provoking, a comparison between participants' self-reported SUDS ratings during the speech task was conducted. There was a significant difference among the three diagnostic groups in SUDS ratings, $F(2,135)=18.00, p<.001(\mathrm{LSD}=.77)$. Means and standard deviations are reported in Table 2. Follow-up analyses were conducted and indicated that the social phobic group reported significantly higher SUDS ratings than the socially anxious and nonanxious groups. Adolescents in the socially anxious group reported significantly higher SUDS ratings than the nonanxious group.

A post hoc power analysis was conducted after group formation to examine the largest pairwise effect size that could reliably be found at the $p=.05$ level while maintaining an acceptable Type II error rate $(\beta=.20)$. The smallest effect size reliably detectable in the current sample, based on the groups just described, was estimated to be between $\eta=.25$ and $\eta=.30$.

\section{Correlational Analyses}

To test the first hypothesis, that social anxiety would be negatively correlated with PA, but not with NA or $\mathrm{PH}$, Pearson's product-moment correlations were computed among the tripartite variables (NA, as measured by the PANAS-N; PA, as measured by the PANAS-P; and $\mathrm{PH}$, as measured by the $\mathrm{PH}$ scale) and social anxiety symptomatology (as measured by the SAS-A and SPAI-C). Correlations among the self-report variables can be found in Table 4. As anticipated, the SAS-A and

Table 2. Means and Standard Deviations on Self-Report Measures by Diagnostic Group

\begin{tabular}{|c|c|c|c|c|c|c|c|c|}
\hline \multirow[b]{2}{*}{ Variable } & \multicolumn{2}{|c|}{ Nonanxious $^{a}$} & \multicolumn{2}{|c|}{ Socially Anxious $^{b}$} & \multicolumn{2}{|c|}{ Socially Phobic ${ }^{c}$} & \multirow[b]{2}{*}{$F$} & \multirow[b]{2}{*}{$\eta$} \\
\hline & $M(S D)$ & $n$ & $M(S D)$ & $n$ & $M(S D)$ & $n$ & & \\
\hline PANAS-N & $17.19_{a}(4.07)$ & 42 & $20.16_{b}(4.98)$ & 56 & $19.19_{b}(5.80)$ & 53 & $4.20^{*}$ & .23 \\
\hline PANAS-P & $34.35_{\mathrm{a}}(5.94)$ & 40 & $34.82_{a}(5.36)$ & 57 & $30.85_{b}(6.47)$ & 52 & $7.00^{* *}$ & .30 \\
\hline PH Scale & $3.78_{a}(3.89)$ & 40 & $5.42_{\mathrm{b}}(3.47)$ & 52 & $6.72_{\mathrm{b}}(4.38)$ & 43 & $5.92^{* *}$ & .29 \\
\hline SAS-A & $40.57_{a}(6.04)$ & 42 & $60.96_{b}(8.53)$ & 57 & $61.96_{b}(14.66)$ & 56 & $58.55^{\star *}$ & .66 \\
\hline SUDS 1 & $.78_{a}(1.15)$ & 41 & $1.89_{b}(1.79)$ & 52 & $2.21_{b}(1.85)$ & 43 & $8.75^{* *}$ & .25 \\
\hline SUDS 2 & $3.41_{a}(1.80)$ & 41 & $4.50_{\mathrm{b}}(1.88)$ & 52 & $5.88_{c}(1.94)$ & 43 & $18.27^{* *}$ & .35 \\
\hline
\end{tabular}

Means with differing subscripts are significantly different from each other by diagnostic group. PANAS = Positive and Negative Affect Scale-Negative scale; Positive and Negative Affect Scale-Positive scale; PH scale = Physiological Hyperarousal scale; SASA = Social Anxiety Scale for Adolescents Total Score; SPAI-C = Social Phobia and Anxiety Inventory for Children Total Score; SUDS 1 = Subjective Units of Distress prior to the speech task; SUDS 2 = Subjective Units of Distress during the speech task.

${ }^{a} n=45 ;{ }^{b} n=57 ;{ }^{c} n=56$

${ }^{*} p<.05 ;{ }^{* *} p<.01$. 
Table 3. Means and Standard Deviations on Heart Rate Reactivity by Diagnostic Group

\begin{tabular}{|c|c|c|c|c|c|c|}
\hline Reactivity $^{d}$ & \multicolumn{2}{|c|}{ Nonanxious $^{a}$} & \multicolumn{2}{|c|}{ Socially Anxious ${ }^{b}$} & \multicolumn{2}{|c|}{ Social Phobic ${ }^{c}$} \\
\hline Minute 2 & $0.93(1.14)$ & 40 & $2.59(1.58)$ & 48 & $2.66(1.48)$ & 41 \\
\hline Minute 3 & $0.42(1.04)$ & 40 & 1.38 (1.67) & 48 & $2.03(1.26)$ & 41 \\
\hline Minute 6 & 1.57 (1.15) & 40 & $1.80(1.43)$ & 46 & 1.89 (1.42) & 40 \\
\hline Minute 7 & $1.30(1.03)$ & 40 & 2.41 (1.37) & 44 & $1.86(1.41)$ & 37 \\
\hline Minute 8 & 1.17 (1.12) & 40 & 2.13 (1.27) & 43 & $0.92(1.49)$ & 36 \\
\hline Minute 9 & 1.64 (1.16) & 40 & $3.20(1.26)$ & 41 & 1.38 (1.57) & 34 \\
\hline
\end{tabular}

${ }^{a} n=45 ;{ }^{b} n=57 ;{ }^{c} n=56 ;{ }^{d}$ All Fs for reactivity at each minute during the speech were nonsignificant.

SPAI-C were significantly negatively correlated with the PANAS-P $(r=-.19, p<.05$ and $r=-.21, p<.05$, respectively). Contrary to our hypotheses results indicated that both the SAS-A and SPAI-C were significantly positively correlated with the PANAS-N $(r=.40$, $p<.01$ and $r=.35, p<.01$, respectively) and the $\mathrm{PH}$ scale $(r=.21, p<.05$ and $r=.28, p<.01$, respectively). Follow-up analyses using Steiger's Z-test of differential predictive strength showed that the SAS-A was significantly more correlated with the PANAS-N than it was with the PANAS-P $(Z=2.12, p<.05)$. The correlations between the SAS-A and PANAS-N and the SAS-A and $\mathrm{PH}$ scale were not significantly different in their predictive strength $(Z=1.70, p>.05)$. Finally, the correlations between the SAS-A and PANAS-P and the SAS$\mathrm{A}$ and $\mathrm{PH}$ scale were also not significantly different in their predictive strength $(Z=.17, p>.05)$. Follow-up analyses using Steiger's Z-test did not show significant differences in predictive strength between the correlations of the SPAI-C and the PANAS-N, PANAS-P, or PH scale.

Table 4. Correlations Among Self-Report Measures

\begin{tabular}{llll}
\hline & PANAS-N & PANAS-P & PH Scale \\
\hline SAS-A & $.40^{* *}$ & $-.19^{*}$ & $.21^{*}$ \\
SPAI-C & $.35^{* *}$ & $-.21^{*}$ & $.28^{* *}$ \\
\hline
\end{tabular}

PANAS-N = Positive and Negative Affect Scale-Negative scale; PANAS-P = Positive and Negative Affect Scale-Positive scale; $\mathrm{PH}$ scale = Physiological Hyperarousal scale; SAS-A = Social Anxiety Scale for Adolescents Total Score; SPAI-C $=$ Social Phobia and Anxiety Inventory for Children Total Score.

${ }^{*} p<.05 ;{ }^{* *} p<.01$

\section{Group Analyses}

Between-groups analyses of variance were conducted to evaluate mean differences in the tripartite constructs of NA, PA, and PH among the three diagnostic groups. Analyses of variance were performed separately for each self-report measure with the three diagnostic groups (i.e., nonanxious, socially anxious, and social phobic) serving as the independent variables and scores on the self-report measures of the tripartite constructs serving as the dependent variables. Follow-up pairwise analyses were calculated using LSD mean minimum differences to determine the cutoff for significant differences. Results (Table 2) indicated that there was a significant mean difference among the groups on the PANAS-P, $F(2,146)=7.00, p<.05, \eta=.30($ LSD $=2.35)$. Contrary to our hypothesis, the social phobic group scored significantly lower than the socially anxious and nonanxious groups. Also contrary to our hypothesis, the socially anxious and nonanxious groups were not significantly different from each other.

Results indicated that there was a significant mean difference among the groups on the PANAS-N, $F(2$, $148)=4.20, p<.05, \eta=.23(\mathrm{LSD}=1.99)$, contrary to our hypothesis. Adolescents in the nonanxious group scored significantly lower than the adolescents classified as socially anxious and social phobic, who did not score significantly different from each other. Results furthermore indicated that, contrary to our hypothesis, there were significant differences among the groups on the $\mathrm{PH}$ scale, $F(2,132)=5.92, p<.01, \eta=.29(\operatorname{LSD}=1.63)$. The nonanxious group scored significantly lower than those in the socially anxious and social phobic groups, who were not significantly different from each other. 
Table 5. Correlations Between Self-Report Measures and Heart Rate Reactivity

\begin{tabular}{lrrrrr}
\hline & SAS-A & SPAI-C & PANAS-N & PANAS-P & PH scale \\
\hline Minute 1 & -.03 & .00 & .01 & .07 & .04 \\
Minute 2 & .04 & .06 & .11 & -.02 & -.07 \\
Minute 3 & -.03 & .04 & .03 & -.01 & -.06 \\
Minute 4 & -.01 & .06 & .04 & -.07 & -.02 \\
Minute 5 & -.04 & .05 & .03 & .04 & -.02 \\
Minute 6 & -.04 & .04 & .04 & .04 & .02 \\
Minute 7 & -.02 & .09 & .03 & .02 & .04 \\
Minute 8 & -.00 & .07 & .04 & .04 & .05 \\
Minute 9 & -.00 & .04 & .08 & .05 & .06 \\
Minute 10 & .04 & .08 & .06 & .03 & .07 \\
\hline
\end{tabular}

SAS-A = Social Anxiety Scale for Adolescents Total Score; SPAI$\mathrm{C}=$ Social Phobia and Anxiety Inventory for Children Total Score; PANAS-N = Positive and Negative Affect Scale-Negative scale; PANAS-P = Positive and Negative Affect Scale-Positive scale; $\mathrm{PH}$ scale $=$ Physiological Hyperarousal scale.

\section{HR Analyses}

To investigate objective $\mathrm{PH}$, correlations were computed among HR reactivity and social anxiety self-reports, as well as HR reactivity and the PH scale. Neither the SAS-A nor the SPAI-C were significantly correlated with HR reactivity during any of the $10 \mathrm{~min}$ of the speech (see Table 5). The PH scale also was not significantly correlated with HR reactivity at any minute during the speech. A between-groups analysis of variance was performed to assess the relationship between diagnostic group and $\mathrm{PH}$ with HR reactivity as the dependent variable and the three diagnostic groups (i.e., nonanxious, socially anxious, and social phobic) serving as the independent variable. Results indicated that there were no significant mean differences among the groups on HR reactivity during any $10 \mathrm{~min}$ of the speech (all Fs were nonsignificant; see Table 3). Also, resting heart rate did not differ between the groups at baseline (all Fs were nonsignificant).

\section{Discussion}

The present study sought to evaluate the relationship of the tripartite constructs to social phobia in a nonclinical sample of adolescents, based on evidence that has suggested differential functioning of the original constructs in social phobia. We expected that social anxiety symptomatology would be negatively correlated with PA but would not be correlated with NA or PH. We also expected that adolescents classified as social phobic would exhibit lower PA compared to socially anxious adolescents, who would exhibit lower PA than nonanxious adolescents, but that there would be no difference among the groups with regard to NA or PH. Last, we examined the relationship between HR reactivity and social anxiety, and HR reactivity and the PH scale.

Social anxiety was negatively correlated with PA, consistent with some research, but contrary to the original tripartite model. Results partially supported the hypothesis that adolescents in the social phobic and socially anxious groups would exhibit lower PA than adolescents in the nonanxious group. Adolescents in the socially anxious group reported comparable PA to adolescents in the nonanxious group but reported higher PA than adolescents in the social phobic group. Thus, self-reported social anxiety symptoms alone are not associated with low PA, but rather the significant distress and impairment associated with social phobia diagnoses are associated with lower PA. Low PA was a significant characteristic of diagnostic social phobia, consistent with research questioning the applicability of the tripartite constructs in social phobia (Brown et al., 1998; Chorpita et al., 2000; Watson, Clark, \& Carey, 1988). However, low PA was not characteristic of social anxiety symptomatology, consistent with the original model.

Other results were consistent with the original model. Contrary to our hypothesis, NA was significantly positively associated with self-reported social anxiety symptomatology. Also contrary to our hypothesis, adolescents in the social phobic and socially anxious groups exhibited higher NA compared to adolescents in the nonanxious group. These results are not in accordance with research that has failed to show NA as a significant component of social phobia (i.e., Chorpita et al., 2000; Watson, Clark, \& Carey, 1988; Watson et al., 2005). Our results support that NA is, in fact, involved in social anxiety and diagnostic social phobia, as predicted by the original model.

With regard to self-reported $\mathrm{PH}$, results also are contrary to our hypothesis. Adolescents in the social phobic and socially anxious groups exhibited higher PH compared to adolescents in the nonanxious group. These results are contrary to research questioning the importance of PH in social phobia (Brown et al., 1998; Chorpita \& Daleiden, 2002; Chorpita et al., 1998; Joiner et al., 1999) but are consistent with the original model. Caution is warranted, however, because the $\mathrm{PH}$ scale contained merely six items from the BAI and may not be a comprehensive measurement of PH. It will be important for future research to use an empirically derived self-report measure of $\mathrm{PH}$, such as the Physiological Hyperarousal Scale for Children (Joiner et al., 1999), which was not available for use at the beginning of data collection for the present study. It also is important to note that the $\mathrm{PH}$ construct was assessed immediately after the speech task and therefore reflects perceived arousal during the speech. Although the $\mathrm{PH}$ scale was demonstrated to have good discriminant validity with subjective anxiety 
(Joiner et al., 1999), this scale was not administered after an anxiety-provoking task in the study by Joiner and colleagues. Therefore, administration of this measure immediately following the speech task may have been contaminated by subjective anxiety ratings. Our results may have been different if the PH scale were administered at the same time as the PANAS and not after the speech task. It would be useful to examine general perceptions of $\mathrm{PH}$, outside of anxiety-provoking situations, given the inadequate definition of the $\mathrm{PH}$ construct in the literature.

Analyses revealed no group differences in objective $\mathrm{PH}$, as measured by HR reactivity, contrary to findings with the $\mathrm{PH}$ scale. The $\mathrm{PH}$ scale was not significantly correlated with any HR reactivity measurements. One reason for this discrepancy may be that the $\mathrm{PH}$ scale measures perceived $\mathrm{PH}$, whereas $\mathrm{HR}$ reactivity is an objective measurement of $\mathrm{PH}$. Thus, the $\mathrm{PH}$ scale and $\mathrm{HR}$ reactivity measure different aspects of the $\mathrm{PH}$ construct. It is possible that perceived $\mathrm{PH}$ is more important to the functioning of social anxiety as proposed in the tripartite model than is objective $\mathrm{PH}$. Alternatively, it is possible that high scores on the PH scale may be a function of increases in state anxiety subsequent to the speech task, which could explain the divergent results with the heart rate data. More research is necessary to better elucidate the relationship between objective and subjective $\mathrm{PH}$ and their relationship to the tripartite construct of $\mathrm{PH}$ as well as to examine the definition of $\mathrm{PH}$ in adolescent social anxiety and social phobia.

Results from the present investigation suggest that all three tripartite constructs are important in the description of social phobia. Adolescents diagnosed with social phobia exhibit low PA, high NA, and high PH, whereas adolescents with high social anxiety but below the threshold for a diagnosis of social phobia evidence high NA and high PH but not low PA. These results suggest that social anxiety symptomatology is consistent with the original tripartite model but support a revised model including low PA as an additional characteristic associated with diagnostic social phobia. The appearance of low PA likely reflects the significant distress and functional impairment related to social phobia diagnoses, but not necessarily with heightened social anxiety. The distress and impairment associated with social phobia may be particularly detrimental to adolescents, given the importance of social activity during this developmental period. Relatedly, as noted, there may be a developmental progression from social phobia to depression (Moutier \& Stein, 1999). It is possible that heightened social anxiety, which in this study fit the predictions of the original tripartite model, progresses toward decreased PA, which is characteristic of depression in the original tripartite model. Lower PA may be related to increased impairment by social anxiety symptoms during adoles- cence, which would lead to a diagnosis of social phobia, and possibly increased depressive symptoms in adulthood.

There are several limitations to the findings of the present investigation. Most important, the generalizability of the present study is limited because the vast majority of adolescents were Caucasian with very little representation of ethnic minorities. It will be important for future research to assess the applicability of the tripartite constructs with culturally and ethnically diverse adolescents who present with social anxiety or social phobia because little research to date has examined cultural and ethnic differences in social phobia. Future research should also examine both the original tripartite model and revised conceptualizations in diverse samples to determine if the models differ based on culture or ethnicity.

Another issue with the present study is the high number of adolescents identified with social phobia. Prevalence rates for social phobia in youth range from $1 \%$ to 3\% (Kashdan \& Herbert, 2001), which is significantly lower than the rate in the present sample. It is important to note, however, that we specifically advertised for adolescents with social anxiety, and therefore our sample was impacted by sampling biases. A related issue is the low incidence of comorbid depression in the current sample. Youth with principal mood disorders did not participate in the study because they were initially screened out, given the purpose of the larger study, which was to study social phobia specifically.

Another concern is that the socially anxious and social phobic groups differed significantly on the SPAI$\mathrm{C}$ but not on the SAS-A. It is possible that this discrepancy results from slightly different constructs being measured by the scales. The SPAI-C was empirically derived and is based on the DSM-IV criteria (Beidel et al., 1995). The SPAI-C assess the impact of different social contexts and is a better indicator of diagnostically significant symptomatology (Inderbitzen-Nolan et al., 2004), whereas the SAS-A was developed to assess avoidance and inhibition (La Greca, 1998) as well as fear of negative evaluation.

There are several weaknesses with the $\mathrm{PH}$ construct in the present study. One constraint is lack of inclusion of measures specifically developed to assess $\mathrm{PH}$, such as the Physiological Hyperarousal Scale for Children (Joiner et al., 1999). In addition, the Affect and Arousal Scale (Chorpita et al., 2000) was developed specifically to assess the tripartite constructs and has shown promising results. Unfortunately, these measures were not available at the beginning of data collection for the present study. Also, the PH scale was administered immediately following the speech task, which raises some concern that ratings were influenced by subjective anxiety. However, Joiner and colleagues (1999) found good discriminant validity for the $\mathrm{PH}$ scale with regard to sub- 
jective anxiety, as measured by SUDS in the current study. Another notable weakness is that heart rate was not monitored or recorded during the 3-min preparatory period prior to the speech. Thus, we were unable to assess whether there were group differences in anticipatory heart rate. Future research should assess arousal not only during an anxiety-provoking task but also prior to the task in order to capture anticipatory anxiety.

The current study is significant because it addresses a number of the serious limitations of previous research. We assessed clinical symptoms through self-reports and a semistructured diagnostic interview. A number of adolescents who did not meet diagnostic criteria for social phobia endorsed a high level of symptoms on one or both of the self-report measures of social anxiety, which suggests that these adolescents experienced significant social anxiety but were not as significantly distressed or impaired by these symptoms. In addition, this group of adolescents fit the predictions of the original tripartite model for anxiety, whereas those with a diagnosis of social phobia did only in part. The inclusion of these groups of adolescents allowed for assessment of differences in the importance of the tripartite constructs across severity levels of social anxiety. Examination of only adolescents diagnosed with social phobia or undiagnosed would have missed the differences in tripartite constructs found in the present study.

Another significant contribution of the present investigation was inclusion of an objective measure of physiological hyperarousal to assess $\mathrm{PH}$. Results from the present investigation failed to find a significant relationship between the $\mathrm{PH}$ scale and $\mathrm{HR}$ reactivity, which suggests a need for more research to better understand the relationship between these objective and subjective measures of PH. This study should encourage future researchers to consider or examine differences in perceived $\mathrm{PH}$ versus objective $\mathrm{PH}$ and to evaluate these two constructs in relation to the tripartite model.

\section{Implications for Research, Policy, and Practice}

Several assessment and treatment implications stem from the present study. Because adolescents diagnosed with social phobia exhibit low PA, it may be important for treatment to address low PA in a manner similar to the treatment of depression. It is possible that this group of adolescents may benefit from the addition of a behavioral activation component to treatment that would assist in elevating affect, which could in turn increase treatment adherence when addressing social fears. Based on the finding that adolescents in the socially anxious and social phobic groups evidenced heightened levels of perceived physiological hyperarousal, it may be important to add a more significant psychoeducational component to treatment regarding physiological responses of anxiety. Such a treatment component is used already in protocols for panic disorder treatment and may be applicable to adolescents with social phobia as well. Assessment of the tripartite constructs would benefit treatment because identification of low PA or high PH could lead to implementation of the aforementioned treatment recommendations. These implications support the creation of different treatment modules, which would allow clinicians to select necessary components based on the symptom presentation of individual adolescents.

In conclusion, findings from the current study support the differential functioning of the tripartite constructs for adolescent social phobia. Findings suggest that all tripartite constructs are necessary in the measurement of social phobia and that social phobia is characterized by low PA, high NA, and high PH. This conceptualization of social phobia is not entirely consistent with Clark and Watson's original tripartite model; however, socially anxious adolescents who did not receive a diagnosis of social phobia did match the pattern (high NA and high PH but not low PA) predicted by the original model. This suggests that the original tripartite model may describe subclinical social anxiety symptoms more accurately than the disorder of social phobia. This study improved upon a number of limitations of previous investigations and investigated an objective measurement of the $\mathrm{PH}$ construct. Findings from this study need to be replicated in ethnically and culturally diverse samples.

Acknowledgments - This research was supported in part by a grant to the third author from the National Institute of Mental Health (R01 MH59608-01A1).

\section{References}

American Psychiatric Association (1994). Diagnostic and statistical manual of mental disorders. American Psychiatric Association, Washington, DC.

Anderson, E. A., and Hope, D. A. (2008). A review of the tripartite model for understanding the link between anxiety and depression in youth. Clinical Psychology Review 28, 276-288.

Beck, A. T., Epstein, N., Brown, G., and Steer, R. A. (1988). An inventory for measuring clinical anxiety: Psychometric properties. Journal of Consulting and Clinical Psychology 56, 893-897.

Beidel, D. C., and Randall, J. Ollendick, T. H., King, N. J., and Yule, W., eds. (1994). Social phobia. International handbook of phobic and anxiety disorders in children and adolescents, pp. 111-129. Plenum, New York.

Beidel, D. C., Turner, S. M., and Fink, C. M. (1996). Assessment of childhood social phobia: Construct, convergent, and discriminative validity of the social phobia and anxiety inventory for children (SPAI-C). Psychological Assessment 8, 235-240. 
Beidel, D. C., Turner, S. M., Hamlin, K., and Morris, T. L. (2000). The social phobia and anxiety inventory for children (SPAI-C): External and discriminative validity. Behavior Therapy 31, 75-87.

Beidel, D. C., Turner, S. M., and Morris, T. L. (1995). A new inventory to assess childhood social anxiety and phobia: The social phobia and anxiety inventory for children. Psychological Assessment 7, 73-79.

Beidel, D. C., Turner, S. M., and Morris, T. L. (1998). Social Phobia and Anxiety Inventory for Children (SPAI-C) manual. Multi-Health Systems, North Tonawanda, NY.

Brady, E. U., and Kendall, P. C. (1992). Comorbidity of anxiety and depression in children and adolescents. Psychological Bulletin 111, 244-255.

Brown, T. A., Chorpita, B. F., and Barlow, D. H. (1998). Structural relationships among dimensions of the anxiety and mood disorders and dimensions of negative affect, positive affect, and autonomic arousal. Journal of Abnormal Psychology 2, 179-192.

Cannon, M. F., and Weems, C. F. (2006). Do anxiety and depression cluster into distinct groups?: A test of the tripartite model predictions in a community sample of youth. Depression and Anxiety 23, 453-460.

Chorpita, B. F., Albano, A. M., and Barlow, D. H. (1998). The structure of negative emotions in a clinical sample of children and adolescents. Journal of Abnormal Psychology 107, 74-85.

Chorpita, B. F., and Daleiden, E. L. (2002). Tripartite dimensions of emotion in a child clinical sample: Measurement strategies and implications for clinical utility. Journal of Consulting and Clinical Psychology 70, 1150-1160.

Chorpita, B. F., Plummer, C. M., and Moffitt, C. E. (2000). Relations of tripartite dimensions of emotion to childhood anxiety and mood disorders. Journal of Abnormal Child Psychology 28, 299-310.

Clark, L. A., and Watson, D. (1991). Tripartite model of anxiety and depression: Psychometric evidence and taxonomic implications. Journal of Abnormal Psychology 100, 316-336.

Cole, D. A., Truglio, R., and Peeke, L. G. (1997). Relation between symptoms of anxiety and depression in children: A multitrait-multimethod-multigroup assessment. Journal of Consulting and Clinical Psychology 65, 110-119.

Crook, K., Beaver, B., and Bell, M. (1998). Anxiety and depression in children: A preliminary examination of the utility of the PANAS-C. Journal of Psychopathology and Behavioral Assessment 20, 333-350.

Epkins, C. E. (2002). A comparison of two self-report measures of children's social anxiety in clinic and community samples. Journal of Clinical Child and Adolescent Psychology 31, 69-79.

Essau, C. A., Conradt, J., and Petermann, F. (1999). Frequency of comorbidity of social phobia and social fears in adolescents. Behaviour Research and Therapy 37, 831-843.

Gerlach, A. L., Wilhelm, F. H., and Roth, W. T. (2003). Embarrassment and social phobia: The role of parasympathetic activation. Journal of Anxiety Disorders 17, 197-210.

Greaves-Lord, K., Ferdinand, R. F., Sondeijker, F. E. P. L., Dietrich, A., Oldehinkel, A. J. Rosmalen, J. G. M., et al. (2007). Testing the tripartite model in young adolescents: Is hyperarousal specific for anxiety and not depression? Journal of Affective Disorders 102, 55-63.
Grossman, P., Wilhelm, F. H., Kawachi, I., and Sparrow, D. (2001). Gender differences in psychophysiological responses to speech stress among older social phobics: Congruence and incongruence between self-evaluative and cardiovascular reactions. Psychosomatic Medicine 63, 765-777.

Heimberg, R. G., Dodge, C. S., Hope, D. A., Kennedy, C. R., and Zollo, L. J. (1990). Cognitive behavioral group treatment for social phobia: Comparison with a credible placebo control. Cognitive Therapy and Research 14, 1-23.

Heimberg, R. G., Hope, D. A., Dodge, C. S., and Becker, R. E. (1990). subtypes of social phobia: Comparison of generalized social phobics and public speaking phobics. The Journal of Nervous and Mental Disease 178, 172-179.

Huebner, E. S., and Dew, T. (1995). Preliminary validation of the positive and negative affect schedule with adolescents. Journal of Psychoeducational Assessment 13, 286-293.

Hughes, A. A., Heimberg, R. G., Coles, M. E., Gibb, B. E., Liebowitz, M. R., and Schneier, F. R. (2006). Relations of the factors of the tripartite model of anxiety and depression to types of social anxiety. Behaviour Research and Therapy 44, 1629-1641.

Inderbitzen, H. M., and Hope, D. A. (1995). Relationship among adolescent reports of social anxiety, anxiety, and depressive symptoms. Journal of Anxiety Disorders 9, 385-396.

Inderbitzen-Nolan, H., Davies, C. A., and McKeon, N. D. (2004). Investigating the construct validity of the SPAI-C: Comparing the sensitivity and specificity of the SPAI-C and the SAS-A. Journal of Anxiety Disorders 18, 547-560.

Inderbitzen-Nolan, H. M., and Walters, K. S. (2000). Social anxiety scale for adolescents: Normative data and further evidence of construct validity. Journal of Clinical Child Psychology 29, 360-371.

Joiner Jr., T. E., Catanzaro, S. J., and Laurent, J. (1996). Tripartite structure of positive and negative affect, depression, and anxiety in child and adolescent psychiatric inpatients. Journal of Abnormal Psychology 105, 401-409.

Joiner Jr., T. E., and Lonigan, C. J. (2000). Tripartite model of depression and anxiety in youth psychiatric inpatients: Relations with diagnostic statues and future symptoms. Journal of Clinical Child Psychology 29, 372-382.

Joiner Jr., T. E., Steer, R. A., Beck, A. T., Schmidt, N. B., Rudd, M. D., and Catanzaro, S. J. (1999). Physiological hyperarousal: Construct validity of a central aspect of the tripartite model of depression and anxiety. Journal of Abnormal Psychology 108, 290-298.

Kashdan, T. B., and Herbert, J. D. (2001). Social anxiety disorder in childhood and adolescence: Current status and future directions. Clinical Child and Family Psychology Review 4, 37-60.

La Greca, A. M. (1998). Manual for the Social Anxiety Scales for Children and Adolescents. University of Miami, Miami, FL.

La Greca, A. M., and Lopez, N. (1998). Social anxiety among adolescents: Linkages with peer relations and friendships. Journal of Abnormal Child Psychology 26, 83-94.

La Greca, A. M., and Stone, W. L. (1993). Social Anxiety Scale for Children-Revised: Factor structure and concurrent validity. Journal of Clinical Child Psychology 22, 17-27.

Last, C. G., Perrin, S., Hersen, M., and Kazdin, A. E. (1992). $D S M-I I I-R$ anxiety disorders in children: Sociodemo- 
graphic and clinical characteristics. Journal of the American Academy of Child and Adolescent Psychiatry 31, 1070-1076.

Last, C. G., Strauss, C. G., and Francis, G. (1987). Comorbidity among childhood anxiety disorders. Journal of Nervous and Mental Disease 175, 726-730.

Laurent, J., and Ettelson, R. (2001). An examination of the tripartite model of anxiety and depression and its application to youth. Clinical Child and Family Psychology Review 4, 209-230.

Lonigan, C. J., Carey, M. P., and Finch Jr., A. J. (1994). Anxiety and depression in children and adolescents: Negative affectivity and the utility of self-reports. Journal of Consulting and Clinical Psychology 62, 1000-1008.

Lonigan, C. J., Hooe, E. S., David, C. F., and Kistner, J. A. (1999). Positive and negative affectivity in children: Confirmatory factor analysis of a two-factor model and its relation to symptoms of anxiety and depression. Journal of Consulting and Clinical Psychology 67, 374-386.

Mauss, I. B., Wilhelm, F. H., and Gross, J. J. (2004). Is there less to social anxiety than meets the eye? Emotion experience, expression, and bodily responding. Cognition and Emotion 18, 631-662.

Morris, T. L., and Masia, C. L. (1998). Psychometric evaluation of the social phobia and anxiety inventory for children: Concurrent validity and normative data. Journal of Clinical Child Psychology 27, 452-458.

Moutier, C. Y., and Stein, M. B. (1999). The history, epidemiology, and differential diagnosis of social anxiety disorder. Journal of Clinical Psychiatry 60, 4-8.

Olino, T. M., Klein, D. N., Lewinsohn, P. M., Rohde, P., and Seeley, J. R. (2007). Longitudinal associations between depressive and anxiety disorders: A comparison of two trait models. Psychological Medicine 38, 353-363.

Phillips, B. M., Lonigan, C. J., Driscoll, K., and Hooe, E. S. (2002). Positive and negative affectivity in children: A multitrait-multimethod investigation. Journal of Clinical Child and Adolescent Psychology 31, 465-479.

Silverman, W. K., and Albano, A. M. (1996). Anxiety Disorders Interview Schedule for DSM-IV: Child version (manual). The Psychological Corporation, San Antonio, TX.
Smith, A. J., Merritt, M. M., and Inderbitzen-Nolan, H. M. (2004, November). Frequently endorsed anxiety provoking situations: A comparison of socially anxious and non-anxious adolescents. Poster presented at the annual meeting for the Association for the Advancement of Behavior Therapy, New Orleans, LA.

Stark, K. D., and Laurent, J. (2001). Joint factor analysis of the children's depression inventory and the revised children's manifest anxiety scale. Journal of Clinical Child Psychology 30, 552-567.

Valentiner, D. P., Gutierrez, P. M., and Blacker, D. (2002). Anxiety measures and their relationship to adolescent suicidal ideation and behavior. Journal of Anxiety Disorders 16, 11-32.

Watson, D., Clark, L. A., and Carey, G. (1988). Positive and negative affectivity and their relation to anxiety and depressive disorders. Journal of Abnormal Psychology 97, 346-353.

Watson, D., Clark, L. A., and Tellegen, A. (1988). Development and validation of brief measures of positive and negative affect: The PANAS scales. Journal of Personality and Social Psychology 54, 1063-1070.

Watson, D., Gamez, W., and Simms, L. J. (2005). Basic dimensions of temperament and their relation to anxiety and depression: A symptom-based perspective. Journal of Research in Personality 39, 46-66.

Weems, C. F., and Stickle, T. R. (2005). Anxiety disorders in childhood: Casting a nomological net. Clinical Child and Family Psychology Review 8, 107-135.

Wittchen, H. -U., Stein, M. B., and Kessler, R. C. (1999). Social fears and social phobia in a community sample of adolescents and young adults: Prevalence, risk factors, and comorbidity. Psychological Medicine 29, 309-323.

Wood, J. J., Piacentini, J. C., Bergman, R. L., McCracken, J., and Barrios, V. (2002). Concurrent validity of the anxiety disorders section of the Anxiety Disorders Interview Schedule for DSM-IV: Child and Parent Versions. Journal of Clinical Child and Adolescent Psychology 31, 335-342. 